\title{
Remains of infection
}

\author{
Alan Barbour \\ Departments of Medicine and Microbiology \& Molecular Genetics, University of California Irvine, Irvine, California, USA.
}

\begin{abstract}
In Lyme disease, musculoskeletal symptoms can persist after treatment, which has led to the hypothesis that the causal organism itself may escape antibiotic therapy. The controversy that surrounds this question extends beyond patients, physicians, and scientists, as public health organizations struggle with how the disease should be diagnosed and treated. Is Lyme disease an infection that resolves, or is the spirochetal agent resilient and evasive? In this issue of the JCI, Bockenstedt et al. address this issue and present compelling evidence that the residues of nonviable spirochetes can persist in cartilaginous tissue long after treatment and may contribute to antibiotic-refractory Lyme arthritis.
\end{abstract}

The Oxford English Dictionary defines "remains" as "parts of a person's body after death; a corpse" (1). We expect this fate for invading bacteria after a course of appropriately chosen, faithfully taken antibiotics and when the patient's immune system is competent. A less common usage for the word is "the survivors of a war, battle, or other destructive event." If the antibiotic course was inadequate in dosage or duration or if the patient had a deficiency in one or more innate or adaptive defenses, survival of some of the bacterial invaders would be no surprise. But what if even under the best of all therapeutic circumstances, bacteria, perhaps in unrecognizable forms, persisted through antibiotic therapy to prosper again, days to months after treatment? When the usual criteria for therapeutic success are met, may there be infection "remains," in the survivorship sense, with a consequence of future pathogenicity? Think of the 1984 movie The Terminator: the assassin robot emerges first from an inferno and then from an explosion, now shorn of integument and limbs but still remorselessly pursuing the heroine. This distinction - between lifeless leftovers and viable hangers-on - is at the heart of a controversy over Lyme disease and one that Bockenstedt and coauthors address in their article in this issue (2).

\section{The controversy}

The controversy in its broadest scope is in its third decade and most simply framed in terms of opinions about diagnostic criteria for Lyme disease and the duration of antibiotic therapy $(3,4)$. Is Lyme disease

Conflict of interest: The author has declared that no conflict of interest exists.

Citation for this article: J Clin Invest. 2012 122(7):2344-2346. doi:10.1172/JCI63975. an infection that resolves, as most bacterial infections do, in the face of an effective immune response, antibiotics, or a combination of the two? Or is the spirochetal agent - Borrelia burgdorferi in North America - unaccountably more resilient and evasive than numerous in vitro and in vivo studies in the laboratory and clinic would have us conclude? The controversy extends beyond academic and public health circles and now encompasses state and federal legislative bodies as well as widespread litigation, including an antitrust suit by Connecticut's attorney general against the Infectious Diseases Society of America (4).

Earlier in the controversy's history, one could roughly characterize the disputants as academic clinicians, microbiologists, and public health epidemiologists on one side and a group of practitioners, mainly without academic affiliations but supported by national and local patient advocacy groups, on the other side. The great majority of papers on antibiotic susceptibilities and therapy protocols in experimental infections and controlled clinical trials bolstered claims of the first group that B. burgdorferi infection, with some exceptions, was cured by courses of single antibiotics within - at most - a few weeks (5). By this account, if some symptoms and disability continued through or recurred after appropriate antibiotic therapy, this plausibly was a postinfection phenomenon, such as Reiter's syndrome and Syndenham's chorea. In the case of B. burgdorferi infection, this was termed "post-Lyme disease syndrome" $(6,7)$. The other side of the dispute generally preferred the term "chronic Lyme disease," with its implication of ongoing infection (8).

There was an occasional paper that reported results that challenged the prevailing view about the spirochete's sus- ceptibility in vitro or in vivo to antibiotics of the $\beta$-lactam, tetracycline, and macrolide classes $(9,10)$. But these provocative reports did not discernibly influence professional opinion makers or alter the research priorities of governmental funding agencies. They did not lead to wider, independent testing of these alternative hypotheses, which by implication entailed lengthier treatments at minimum and radically different therapies at the extreme. Those advocating unconventional treatment measures continue to cite as justification the evidence from a handful of subsequently published articles, but, in my view, most investigators in the Lyme disease research field did not attach much credence to these reports. If this was complacency, it was shaken by unsettling reports from three research groups, who from a partisan's view, were among the academic and public health "establishment."

\section{Questioning convention}

In 2002, Bockenstedt et al. reported the persistence of B. burgdorferi DNA in tissues and its uptake into xenodiagnostic ticks feeding on mice that had been treated for infection several weeks to months previously (11). The authors concluded that whatever was persisting in the mouse and was transmitted from mouse to tick (though no further) was not infectious in the usual sense and called these spirochetes "attenuated." This interpretation recalled publications from the 1960s - the heyday of attention on posttreatment "L-forms" and other cell-walldeficient bacteria and their possible association with recurrent or chronic disease (reviewed in ref. 12). Hodzic et al. reported a similar duration of PCR positivity of tissues after treatment in experimental infections of mice but also went further in claiming that some of these "nondividing" spirochetes were infectious and transmissible beyond the tick vector to naive mice on which the ticks subsequently fed (13). These articles were cited by Stricker and Johnson in their editorial defense of the concept of chronic infection as the cause of persistent symptoms after standard therapeutic measures (14). Wormser and 


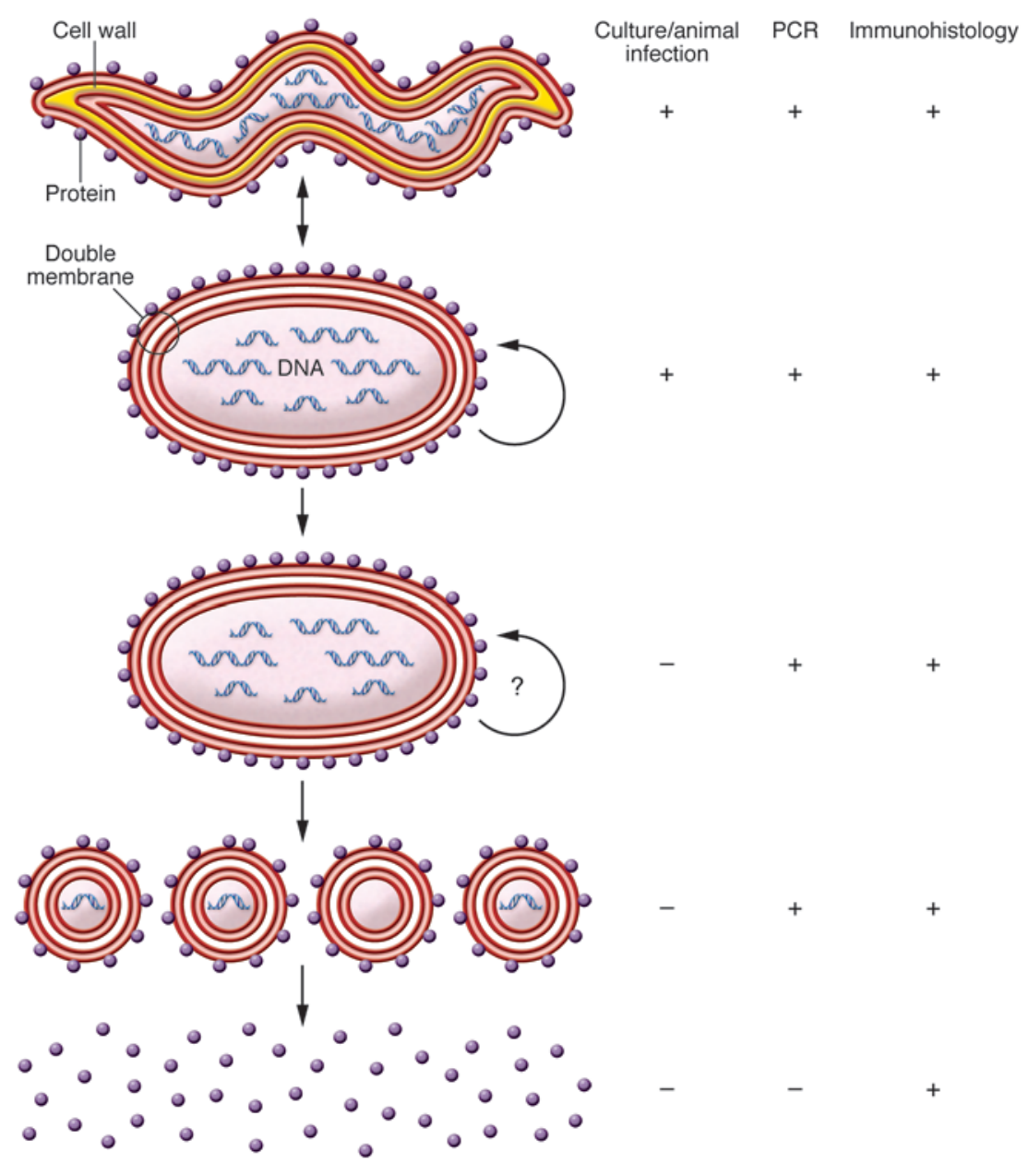

Figure 1

Detectable remains of spirochete infection. Following antibiotic treatment and host immune attack, an intact, replicating spirochete may descend to a state where only proteins of the bacteria remain detectable in the host tissue. The first transition is from the effect of a cell wall-active antibiotic. This can result in some cells that don't have cell walls (also called L-forms or protoplasts) and, consequently, are not affected by the antibiotic anymore, but can still replicate (the circular arrow indicates replication of that form of cell). These cells can revert to normal bacteria with cell walls in the absence of the antibiotic. The next step down are intact cells that contain DNA and protein, but that cannot revert and may or not be able to replicate. Then there is autolysis or immune-mediated breakup of the bacteria into vesicles that contain both DNA and protein or protein alone (under these conditions, free DNA would be rapidly degraded by nucleases). Finally, with the destruction or turnover of the bacterial nucleic acid, only protein remains. The cells become spherical or pleomorphic in the absence of cell wall.

Schwartz critiqued parts of the experimental designs and the interpretation of the results in these two reports (15). In a subsequent article, Barthold et al. rebutted these criticisms and reported additional evidence that "noncultivable" spirochetes in the blood and tissues of treated mice were infectious on the basis of transplantation of tissue allografts and their transmission to naive mice via vector ticks (16). More recently, a paper by Embers et al. added more fuel to the fire by concluding on the basis of xenodiagnosis that " $B$. burgdorferi can withstand antibiotic treatment, administered post-dissemination, in a primate host" (17).

\section{What's left behind}

In their article in this issue of the JCI, Bockenstedt et al. recognize the far-reaching consequences of claims about persistent viability and use different approaches, including culture, tissue transplantation, and xenodiagnosis, to assess this possibility. They come down on the side of the existence of nonviable and nontransmis- sible posttreatment spirochetes in their mouse model. But more important were the experiments using real-time imaging of transgenic fluorescent B. burgdorferi in mice and the transplantation of cartilaginous tissue to naive mice to assess antigenicity and mitogenicity (2). The results of these latter experiments provide compelling evidence that there are residues of the bacteria in the form of protein if not transcribable DNA continuing to cause mischief long after bacterial life has ceased. The authors, with justification, propose that this accounts for the phenomenon of antibiotic-refractory Lyme arthritis as well perhaps as less distinctive musculoskeletal manifestations after treatment.

One conceptualization of the different possible states between a viable, proliferating bacterium and its total absence as well as the predicted outcomes of the different detection measures is shown in Figure 1. This depicts a spirochete and a cell-wallactive antibiotic, but would apply to other types of bacteria and, in modified form, to antibiotics that have other targets. The spirochete shown missing a cell wall may replicate in this state and revert to the usual morphology corresponding to descriptions of L-forms. Beyond this state, there may possibly be replication, but neither reversion nor the possibility of transmission. Finally there are membrane blebs, which may or may not contain nucleic acids, and then only proteins. Either of the last two states could be what Bockenstedt et al. observed (2).

\section{A wider angle view}

Drawing back from the close-up shot on this Lyme disease controversy, can we see connections in the background with other infectious diseases and their posttreatment outcomes? Would these in-depth studies of persistence of pathogen viability and residual DNA and proteins in a host have arisen in the absence of a medical and social controversy that inspired Congressional investigations, advocacy and counter groups among lay individuals and practitioners, picketers at medical meetings, and numerous lawsuits? In my view, yes. I think that the nosology of post-Lyme disease syndrome has utility and that investigations along the lines of the work described here will yield further insights not just about Lyme disease but about other postinfection sequelae, including reactive arthritis, some forms of chronic fatigue syndrome, and perhaps other disorders yet to be rec- 
ognized. While the authors' findings on experimental infections of laboratory mice may not change opinions on appropriate antibiotic therapy for patients, they point to testable hypotheses on the mechanisms for lingering illness after treatment of infection $(18,19)$ and laboratory means to identify persistent antigenic and mitogenic stimulation.

With PCR and sensitive antigen detection methods so readily at hand, we may neglect more direct but time-consuming and artful gauges of viability. Almost 50 years ago, Gutman, Turck, Petersdorf, and Wedgwood reported in the JCI the survival of bacterial variants in antibiotic-treated patients with pyelonephritis (20). They used painstaking methods in a pre-PCR microbiology lab for "separation of bacterial variants from classical organisms.” These remains in the urine were uncultivable by routine procedures but, according to the authors, lived to cause disease again.

Address correspondence to: Alan Barbour, Departments of Medicine and Microbiology \& Molecular Genetics, University of California Irvine, 3012 Hewitt Hall, Irvine, California 92697-4028, USA. Phone:
949.824.5626; Fax: 949.824.8598; E-mail: abarbour@uci.edu.

1. Simpson J, Weiner E, eds. Oxford English Dictionary. 2nd ed. Oxford, United Kingdom: Oxford University Press; 1989.

2. Bockenstedt LK, Gonzalez DG, Haberman AM, Belperron AA. Spirochete antigens persist near cartilage after murine Lyme borreliosis therapy. J Clin Invest. 2012;122(7):2652-2660.

3. Barbour AG, Fish D. The biological and social phenomenon of Lyme disease. Science. 1993;260(5114):1610-1616.

4. Tonks A. Lyme wars. BMJ. 2007;335(7626):910-912.

5. Wormser GP, et al. The clinical assessment, treatment, and prevention of lyme disease, human granulocytic anaplasmosis, and babesiosis: clinical practice guidelines by the Infectious Diseases Society of America. Clin Infect Dis. 2006;43(9):1089-1134.

6. Feder HM Jr, Johnson BJ, O'Connell S, Shapiro ED, Steere AC, Wormser GP. A critical appraisal of "chronic Lyme disease". N Engl J Med. 2007;357(14):1422-1430.

7. Marques A. Chronic Lyme disease: a review. Infect Dis Clin North Am. 2008;22(2):341-360.

8. Cameron D, et al. Evidence-based guidelines for the management of Lyme disease. Expert Rev Anti Infect Ther. 2004;2(1 suppl):S1-S13.

9. Brorson O, Brorson SH. An in vitro study of the susceptibility of mobile and cystic forms of Borrelia burgdorferi to metronidazole. APMIS. 1999;107(6):566-576.

10. Straubinger RK, Summers BA, Chang YF, Appel MJ. Persistence of Borrelia burgdorferi in experimentally infected dogs after antibiotic treatment. J Clin Microbiol. 1997;35(1):111-116.
11. Bockenstedt LK, Mao J, Hodzic E, Barthold SW, Fish D. Detection of attenuated, noninfectious spirochetes in Borrelia burgdorferi-infected mice after antibiotic treatment. J Infect Dis. 2002;186(10):1430-1437.

12. Domingue GJ Sr, Woody HB. Bacterial persistence and expression of disease. Clin Microbiol Rev. 1997;10(2):320-344.

13. Hodzic E, Feng S, Holden K, Freet KJ, Barthold SW. Persistence of Borrelia burgdorferi following antibiotic treatment in mice. Antimicrob Agents Chemother. 2008;52(5):1728-1736.

14. Stricker RB, Johnson L. Lyme disease: the next decade. Infect Drug Resist. 2011;4:1-9.

15. Wormser GP, Schwartz I. Antibiotic treatment of animals infected with Borrelia burgdorferi. Clin Microbiol Rev. 2009;22(3):387-395.

16. Barthold SW, Hodzic E, Imai DM, Feng S, Yang $\mathrm{X}$, Luft BJ. Ineffectiveness of tigecycline against persistent Borrelia burgdorferi. Antimicrob Agents Chemother. 2010;54(2):643-651.

17. Embers ME, et al. Persistence of Borrelia burgdorferi in rhesus macaques following antibiotic treatment of disseminated infection. PloS One. 2012;7(1):e29914.

18. Steere AC, Drouin EE, Glickstein LJ. Relationship between immunity to Borrelia burgdorferi outersurface protein A (OspA) and Lyme arthritis. Clin Infect Dis. 2011;52(suppl 3):s259-s265.

19. Wormser GP, Nadelman RB, Schwartz I. The amber theory of Lyme arthritis: initial description and clinical implications [published online ahead of print March 13, 2012]. Clin Rheumatol. doi:10.1007/ s10067-012-1964-x.

20. Gutman LT, Turck M, Petersdorf RG, Wedgwood RJ. Significance of bacterial variants in urine of patients with chronic bacteriuria. J Clin Invest. 1965;44(12):1945-1952

\title{
What's in a name?
}

\author{
Mitchell J. Weiss, Philip J. Mason, and Monica Bessler
}

The Children's Hospital of Philadelphia, Division of Hematology, Philadelphia, Pennsylvania, USA.

\begin{abstract}
Mutations in numerous genes encoding ribosomal proteins (RPs) occur in $50 \%-70 \%$ of individuals with Diamond-Blackfan anemia (DBA), establishing the disease as a ribosomopathy. As described in this issue of JCI, Sankaran, Gazda, and colleagues used genome-wide exome sequencing to study DBA patients with no detectable mutations in RP genes. They identified two unrelated pedigrees in which the disease is associated with mutations in GATA1, which encodes an essential hematopoietic transcription factor with no known mechanistic links to ribosomes. These findings ignite an interesting and potentially emotional debate on how we define DBA and whether the term should be restricted to pure ribosomopathies. More generally, the work reflects the powerful knowledge and controversies arising from the deluge of data generated by new genetic technologies that are being used to analyze human diseases.
\end{abstract}

\section{The history of DBA}

In 1938, pediatricians Louis Diamond and Kenneth Blackfan described a congenital anemia with hypoplasia of red blood cell

Conflict of interest: The authors have declared that no conflict of interest exists.

Citation for this article: J Clin Invest. 2012; 122(7):2346-2349. doi:10.1172/JCI63989. precursors and concomitant congenital extrahematopoietic anomalies in about one-third of patients (1). The etiology of this syndrome, now known as DiamondBlackfan anemia (DBA), has fascinated and perplexed pediatric hematologists for many years. In 1997, Dahl and colleagues identified a child with DBA and a X:19 chromosomal translocation, linking a critical region of chromosome 19 to DBA in a proportion of multiplex families (2). Positional cloning revealed that the mutated gene was RPS19, which encodes a protein component of the small 40S ribosomal subunit (3). Subsequently, RPS19 mutations were identified in approximately $25 \%$ of DBA families, all of which showed dominant inheritance. Speculation about how RPS19 mutations might cause DBA ensued for about 10 years. Specifically, it was debated as to whether the disease results from loss of unique extraribosomal activities of RPS19 or through impaired ribosome production. Support for the latter hypothesis emerged when a flurry of other DBA genes were identified, all of which encoded different small or large ribosomal subunit proteins (RPs) (4).

\section{DBA perceived as a ribosomopathy}

Currently, $50 \%-70 \%$ of DBA patients are accounted for by mutations in one of 